\title{
Nondestructive beam energy-spread monitor using multi-strip-line electrodes
}

\author{
T. Suwada, ${ }^{*}$ M. Satoh, and K. Furukawa \\ Accelerator Laboratory, High Energy Accelerator Research Organization (KEK), 1-1 Oho, Tsukuba, Ibaraki 305-0801, Japan
}

(Received 29 January 2003; published 27 March 2003)

\begin{abstract}
A nondestructive beam energy-spread monitor using multi-strip-line electrodes has been newly developed in order to measure and control the energy spread of a single-bunch electron beam at an $180^{\circ}$ arc with a beam energy of $1.7 \mathrm{GeV}$ in the KEKB injector linac. The detection principle is described based on a multipole-moment analysis of an electromagnetic field generated by a charged beam. A proof-of-principle experiment was performed to verify the analysis. The result shows that taking into account the second-order moment derived from the multipole-moment analysis, the analyzed transverse beamwidths are consistent with those measured by a screen monitor, and the energy spreads are estimated to be $0.150 \% \pm 0.007 \%$ and $0.264 \% \pm 0.004 \%$ for electron beams with charges of 0.9 and $8 \mathrm{nC} /$ bunch at the rf phase of the energy-spread minimum, respectively. In this report we describe in detail the experimental result using the beam energy-spread monitor along with a theoretical multipolemoment analysis.
\end{abstract}

DOI: 10.1103/PhysRevSTAB.6.032801

PACS numbers: 29.17.+w, 41.75.Ht, 41.85.Ew, 41.85.Qg

\section{INTRODUCTION}

The KEK B-Factory (KEKB) project [1] is in progress for testing $C P$ violation in the decay of $\mathrm{B}$ mesons. KEKB is an asymmetric electron-positron collider comprising $3.5-\mathrm{GeV}$ positron and $8-\mathrm{GeV}$ electron rings. The KEKB injector linac [2] injects single-bunch positron and electron beams directly into the KEKB rings. The beam charges are designed to be of 0.64 and $1.3 \mathrm{nC} /$ bunch, with a maximum repetition rate of $50 \mathrm{~Hz}$, for the positron and electron beams, respectively. High-current primary electron beams $(\sim 10 \mathrm{nC} /$ bunch $)$ are required in order to generate sufficient positrons. Since KEKB is a factory machine, a well-controlled operation of the injector linac is required for keeping the injection rate as high as possible and for maintaining stable operation. For this purpose, beam diagnostic and monitoring tools are essential. Approximately 90 strip-line-type beam-position monitors (BPMs) [3] are utilized to keep the beam orbits stable; in particular, the transverse beam positions of the primary high-current electron beams must be controlled in order to suppress transverse wakefields. Stable control of the beam positions and energies through the beamposition and energy-feedback systems [4] is essential in daily operation. It is also important to acquire beam characteristics of two beam bunches separated by $96 \mathrm{~ns}$ in an rf pulse in which scheme the two beam bunches are injected to the KEKB rings simultaneously [5]. The spatial beam profile is usually measured using fluorescent screen monitors [6] and wire scanners [7]. These monitors are used in measuring the beam optics and in carrying out optics matching along the linac. However, these monitors have several drawbacks in daily operation because the screen monitors destroy the beam itself, and the wire

*Electronic address: tsuyoshi.suwada@kek.jp. scanner cannot obtain any beam sizes pulse by pulse, although they can measure precise transverse beam sizes by detecting high-energy $\gamma$ rays generated from thin wires.

Pioneering work by Miller et al. [8] showed that a stripline-type BPM with four electromagnetic pickups could be utilized as a nonintercepting emittance monitor by theoretically developing a multipole-moment analysis of an electromagnetic field generated by a charged beam in the BPM. They also experimentally demonstrated that transverse emittances of electron beams could be derived from the second-order moment of the electromagnetic field. Our previous work [9] using similar strip-linetype BPMs clearly showed that the second- and thirdorder moments, depending upon the transverse beam sizes, were directly measured by changing the field strength of a quadrupole magnet. Such experimental demonstrations show that the strip-line-type BPMs are applicable for the transverse beam-size measurements pulse by pulse.

A nondestructive beam energy-spread monitor (BESM) using multi-strip-line electrodes has been newly developed in order to measure and control the energy spread of an electron beam in the KEKB injector linac. The energy spread of the primary electron beam for positron generation is often increased due to a longterm phase drift of high-power and booster klystrons. Thus, beam diagnostic and monitoring tools are also required to cure the beam-energy spread; furthermore, those are expected to control the longitudinal wakefields of the high-current primary electron beam pulse by pulse, especially at the $180^{\circ}$ arc of the injector linac. The BESM is one of the very useful monitoring tools for satisfying such requirements, since it can measure the energy spread derived from a quadrupole moment of an electron beam. This monitor was designed based on a numerical analysis 
by applying the multipole moments of the electromagnetic field generated by a charged beam [10]. The BESM directly detects the spread of transverse beamwidths at a large energy dispersion section by measuring any variation of the electromagnetic field distribution induced on the multi-strip-line electrodes of the monitor. In this report the authors not only present a clear experimental verification of this analysis based on the previous work, but also demonstrate that the second-order moment of an electron beam can be nondestructively measured by the BESM, depending on the energy spread at the large energy dispersion section.

\section{MULTIPOLE-MOMENT ANALYSIS OF A CHARGED BEAM}

The electromagnetic field generated by a relativistic charged beam inside a conducting duct is predominantly boosted in the transverse direction to the beam axis due to Lorentz contraction. This phenomenon shows that if the wall loss of the image charges is negligibly small, the electromagnetic coupling of the inner surface of the duct to the beams can be well treated as a two-dimensional electrostatic potential problem. Thus, a derivation of the image charges induced on the duct is simply attributed to the electrostatic potential problem on the transverse plane. For a conducting round duct, the image charges induced by a line charge can be solved as a boundary problem in which the electrostatic potential is equal on the duct [11]. The formula for the image charge density $j$ is given by

$$
j(r, \phi, R, \theta)=\frac{I(r, \phi)}{2 \pi R} \frac{R^{2}-r^{2}}{R^{2}+r^{2}-2 r R \cos (\theta-\phi)},
$$

where $I$ is the line charge, $(r, \phi)$ and $(R, \theta)$ are the polar coordinates of the line charge and the pickup point on the duct, respectively, and $R$ is the duct radius (see Fig. 1).

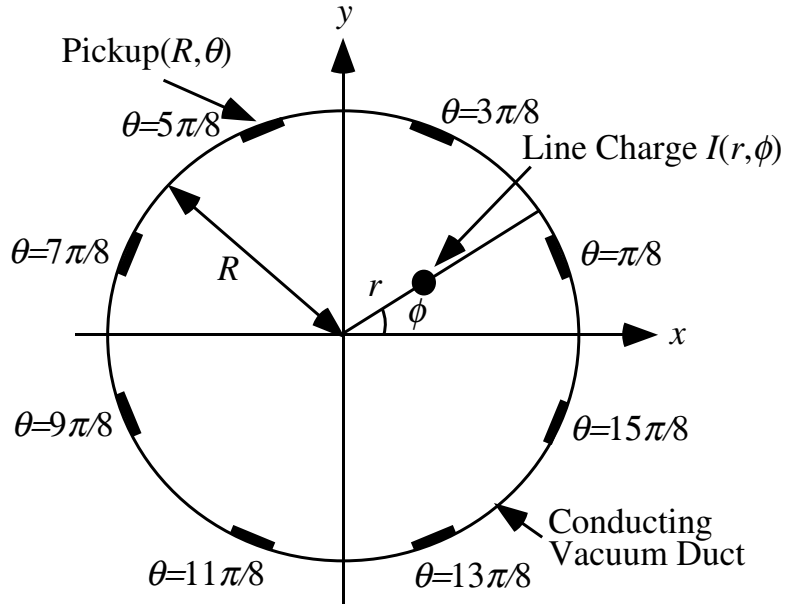

FIG. 1. Polar coordinates of a line charge and eight pickups defined in a conducting duct.

The formula is also represented by an expansion in a power series of $r / R$ according to a similar treatment by Miller et al. [8],

$$
j(r, \phi, R, \theta)=\frac{I(r, \phi)}{2 \pi R}\left[1+2 \sum_{n=1}^{\infty}\left(\frac{r}{R}\right)^{n} \cos n(\theta-\phi)\right] .
$$

Assuming the transverse $r$ distribution $\rho(r)$ of a traveling charged beam, the total image charge $J$ is formulated by integrating the image charge density with a weight of the transverse distribution inside the duct area,

$$
J(R, \theta)=\int_{0}^{R} \int_{0}^{2 \pi} j(r, \phi, R, \theta) \rho(r) r d r d \phi
$$

It is easily expanded by a power series,

$$
J(R, \theta)=\frac{I_{b}}{2 \pi R}\left\{1+\frac{2}{R}(\langle x\rangle \cos \theta+\langle y\rangle \sin \theta)+\frac{2}{R^{2}}\left[\left(\left\langle x^{2}\right\rangle-\left\langle y^{2}\right\rangle+\langle x\rangle^{2}-\langle y\rangle^{2}\right) \cos 2 \theta+2(\langle x y\rangle+\langle x\rangle\langle y\rangle) \sin 2 \theta\right]+\text { higher orders }\right\},
$$

where $I_{b}$ is the beam charge, $\langle x\rangle$ and $\langle y\rangle$ are the charge centers of gravity of the beam, $\left\langle x^{2}\right\rangle$ and $\left\langle y^{2}\right\rangle$ are the horizontal and vertical mean square half-widths of the beam in the $x$ and $y$ directions, and $\langle x y\rangle$ is the $x-y$ coupling in the transverse plane, respectively. The expansion of the integration is well approximated in the case where the transverse beamwidths and positions are small compared to the duct radius. The first to fourth expanded terms correspond to the monopole, dipole (first-order) moment, quadrupole (second-order) moment, and higher-order moments, respectively. These multipole moments are defined in rectangular coordinates as follows:

$$
\langle x\rangle=\iint x j(x, y) \rho(x, y) d x d y,
$$

$$
\begin{aligned}
\langle y\rangle & =\iint y j(x, y) \rho(x, y) d x d y, \\
\left\langle x^{2}\right\rangle & =\iint x^{2} j(x, y) \rho(x, y) d x d y, \\
\left\langle y^{2}\right\rangle & =\iint y^{2} j(x, y) \rho(x, y) d x d y, \\
\langle x y\rangle & =\iint x y j(x, y) \rho(x, y) d x d y .
\end{aligned}
$$

A beam-position measurement is, thus, performed by 
detecting the dipole moment $J_{d}$ of the beam, and a transverse beamwidth measurement is performed to detect the quadrupole moment $J_{q}$ at the least orders. The skew angle of the beam in the transverse plane is also derived from the quadrupole moment and the skew moment $J_{s}$ at the least orders. These multipole moments are described by using the following eight-pickup amplitudes $\left[V_{i}(i=\right.$ 1-8)] of the BESM:

$$
\begin{aligned}
& J_{d_{x}} \equiv \frac{\langle x\rangle}{R}=\int_{0}^{2 \pi} J(R, \theta) \cos \theta d \theta / \int_{0}^{2 \pi} J(R, \theta) d \theta \\
& \simeq \frac{\sum_{i=1}^{8} V_{i} \cos \theta}{\sum_{i=1}^{8} V_{i}} \\
& J_{d_{y}} \equiv \frac{\langle y\rangle}{R}=\int_{0}^{2 \pi} J(R, \theta) \sin \theta d \theta / \int_{0}^{2 \pi} J(R, \theta) d \theta \\
& \simeq \frac{\sum_{i=1}^{8} V_{i} \sin \theta}{\sum_{i=1}^{8} V_{i}} \\
& J_{q} \equiv \int_{0}^{2 \pi} J(R, \theta) \cos 2 \theta d \theta / \int_{0}^{2 \pi} J(R, \theta) d \theta \\
&=\frac{1}{R^{2}}\left(\left\langle x^{2}\right\rangle-\left\langle y^{2}\right\rangle+\langle x\rangle^{2}-\langle y\rangle^{2}\right) \\
& \simeq \frac{\sum_{i=1}^{8} V_{i} \cos 2 \theta}{\sum_{i=1}^{8} V_{i}} \\
& J_{s} \equiv \int_{0}^{2 \pi} J(R, \theta) \sin 2 \theta d \theta / \int_{0}^{2 \pi} J(R, \theta) d \theta \\
&=\frac{2}{R^{2}}(\langle x y\rangle+\langle x\rangle\langle y\rangle) \\
& \simeq \frac{\sum_{i=1}^{8} V_{i} \sin 2 \theta}{\sum_{i=1}^{8} V_{i}} .
\end{aligned}
$$

Normalization by summing the eight-pickup amplitudes needs to cancel out the beam charge fluctuations due to any beam measurement jitter. It is understood that the quadrupole moment is related to the transverse beamwidths, while it also depends on the beam positions. It is noted that the absolute beamwidths cannot be independently obtained by the BESM, because only the dif- ference of the squared beamwidths is related to the quadrupole moment. This is because the equipotential lines are invariant under the condition $\left\langle x^{2}\right\rangle-\left\langle y^{2}\right\rangle=$ const if the beam positions do not change.

\section{BEAM MONITORS}

\section{A. Beam energy-spread monitor}

Figures 2(a) and 2(b) show schematic cross-sectional drawings of the BESM. The mechanical design structure is briefly summarized. The BESM is a conventional monitor with eight-strip-line electrodes fabricated from stainless steel (SUS304) with $\pi / 8$ rotational symmetry. The strip-line length $L$ was determined to be as long as could possibly be installed into the limited spaces in the beam line so as to increase the signal-to-noise ratio. The inner and outer radii, $R_{1}$ and $R_{2}$, and the angular width $\alpha$ of the electrode were chosen so as to characterize a $50-\Omega$-transmission line. The eight pickups with a relatively narrow angular width of $15^{\circ}$ are mounted with a tilt of $\pi / 8 \mathrm{rad}$ at the symmetrical polar coordinates in order to avoid any direct impinging of synchrotron radiation and off-energy electrons to the electrode surfaces in a large energy dispersion section. A $50-\Omega$-vacuum-feedthrough is connected to the upstream side of each electrode, while the downstream end is shortcircuited to a vacuum duct in order to simplify the mechanical manufacturing process. Quick-release flange couplings (NW50, standard KF flange) are used at each end of the monitor for easy installation into the beam line. The mechanical design parameters are summarized in Table I.

\section{B. Screen monitor}

A screen-monitor system (SC) [6] with a screen material $\left(99.5 \% \mathrm{Al}_{2} \mathrm{O}_{3}+0.5 \% \mathrm{CrO}_{3}\right)$ was used to calibrate the transverse beamwidths. This system consists of a fluorescent screen monitor viewed by a high-resolution beamsynchronous charge-coupled device (CCD) camera and a video-image frame grabber installed on a Versa Module Europa-based computer. The beam-profile image from the
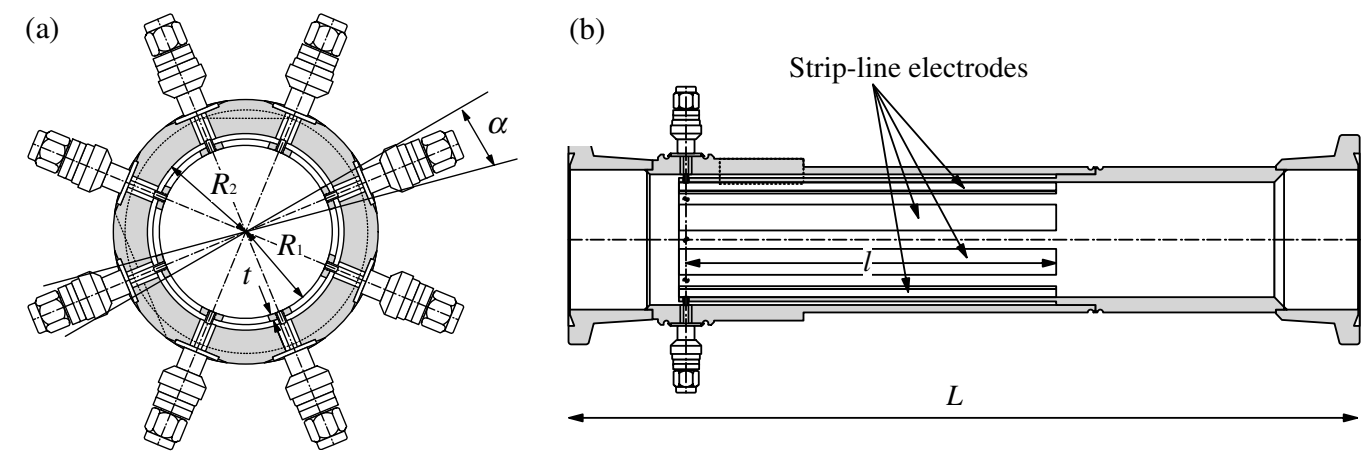

FIG. 2. Schematic cross-sectional drawings [(a) front and (b) side views] of the beam energy-spread monitor. 
TABLE I. Mechanical design parameters of the BESM.

\begin{tabular}{lc}
\hline \hline \multicolumn{1}{c}{ Mechanical parameter } & \\
\hline Innner radius $R_{1}(\mathrm{~mm})$ & 20.6 \\
Outer radius $R_{2}(\mathrm{~mm})$ & 23.4 \\
Electrode angular width $\alpha(\mathrm{deg})$ & 15 \\
Electrode thickness $t(\mathrm{~mm})$ & 1.5 \\
Electrode length $l(\mathrm{~mm})$ & 132.5 \\
Total length $L(\mathrm{~mm})$ & 283 \\
\hline \hline
\end{tabular}

screen monitor is sent out from the CCD camera to the frame grabber as a video-image analog signal. The signal is digitized and stored in the video memory in 512 (vertical) $\times 512$ (horizontal) pixels with a gradation of 8 bit. The UNIX-based host computer analyzes the image data to obtain the spatial beamwidths in half-width at half maximum (HWHM) in the horizontal and vertical directions; the results can be displayed on an $X$ terminal. The spatial resolution of the beam profile is about 4 pixels/mm by using some optical expansion lenses in front of the CCD camera. The spatial resolution is sufficient to measure the transverse beamwidths in this experiment.

\section{EXPERIMENTAL SETUP}

\section{A. Beam line and experimental setup}

Figure 3 shows the schematic layout of the beam line up to sector 1 and the experimental setup.

Single-bunch electron beams can be generated by a new preinjector [12], which comprises two subharmonic bunchers, a prebuncher and a buncher. The electron gun can generate a beam charge of about $20 \mathrm{nC} /$ pulse with a maximum repetition rate of $50 \mathrm{~Hz}$; for positron production, single-bunch electron beams greater than $10 \mathrm{nC} /$ bunch can be stably accelerated from the outlet of the buncher up to a positron-production target installed in sector 2. On the other hand, a single-bunch electron beam of about $1 \mathrm{nC} /$ bunch can be directly accelerated up to $8 \mathrm{GeV}$ at the end of the injector linac. The nominal beam energies are about $500 \mathrm{MeV}$ and $1.7 \mathrm{GeV}$ at the end of sectors A and B, respectively. A longitudinal beam profile can be monitored using an optical transition-radiation monitor (OTR) with a streak-camera system [13] installed after the exit of module A-1. Beam experiments using electron beams of 0.9 and $8 \mathrm{nC} /$ bunch were carried out at the $180^{\circ}$ arc after sector B. Here, the transport system of the arc is briefly summarized, since a detailed design report is available elsewhere [14]. The transport system was designed to be achromatic and isochronous in order to preserve the emittance and bunch time structure during passage of the electron beam through the arc. The arc section is composed of six $30^{\circ}$ bending magnets, seven quadrupole magnets, and six sextupoles. Seven strip-line-type BPMs are installed inside each quadrupole for the beam orbit and dispersion measurement. The BESM was installed inside a sextupole at the center of the arc, and the screen monitor used in this experiment was installed $350 \mathrm{~mm}$ behind the BESM. The multipole moments of the electron beam were measured by the BESM while the spatial beamwidths were directly measured by the screen-monitor system simultaneously. The transverse beamwidths at the locations of these monitors were controlled by changing the rf phase of the booster klystron at sector B. The BPMs located at sectors A, B and the arc monitored the beam positions and charges of the beam in order to control the beam positions and energy stably through the feedback systems without any beam loss during the experiment.

\section{B. Data-acquisition system}

The data-acquisition system of the BESM comprises a signal-digitizing system of an oscilloscope with a sampling rate of $8 \mathrm{GS} / \mathrm{s}$ and a bandwidth of $1 \mathrm{GHz}$, which is controlled by a PC/Linux-based computer with a Pentium IV microprocessor at $2.2 \mathrm{GHz}$. The eight signals of the monitor are divided by two signal groups

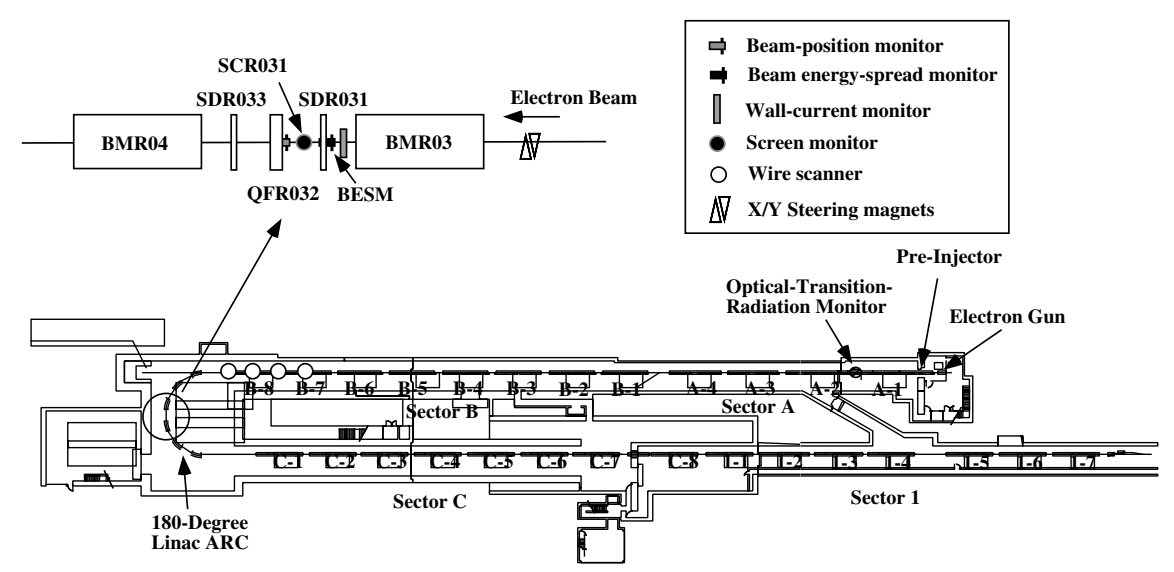

FIG. 3. Schematic layout of the beam line up to sector 1 and the experimental setup. 
alternately $(2 \times 4$ signals $)$. The four signals of each signal group are sent directly to a signal combiner through 35$\mathrm{m}$-long coaxial cables with suitable delay lines, which are required to avoid a superposition of the analog signals. The two combined signals out of the signal combiners are directly connected to the digital oscilloscope. Trigger pulses synchronized with the beam are provided to the oscilloscope at a maximum rate of $50 \mathrm{~Hz}$ cycle. This rate is applicable for communication between the computer and the oscilloscope through an ethernet. The computer receives digitized data from the oscilloscope, extracts the eight-pickup voltages, and calculates the beam positions, charge, and the multipole moments pulse by pulse in $50 \mathrm{~Hz}$. It is even possible to observe two beam bunches separated $96 \mathrm{~ns}$ in an $\mathrm{rf}$ pulse. The analyzed data are displayed on any $X$ terminals through an application program.

\section{BEAM TEST}

A beam test was carried out by using single-bunch electron beams with charges of 0.9 and $8 \mathrm{nC} /$ bunch at an energy of $1.7 \mathrm{GeV}$ under the nominal operation condition. Typical longitudinal profiles were measured by the OTR, and the bunch lengths for both electron beams were $12 \mathrm{ps}$ in full width at half maximum. The maximum repetition rate was chosen to be $25 \mathrm{~Hz}$ in order to reduce the radiation damage of the screen monitor.

First of all, the beam experiments with the BESM were performed by measuring the quadrupole moment of the 0.9 -nC electron beam, as a function of the horizontal $(x)$ and vertical $(y)$ beam positions. In this measurement the rf phase of the booster klystron at sector B was fixed to reduce the energy spread to its minimum with the beamorbit and energy feedbacks at sector B off. The beam positions at the BESM were controlled by the field strength of upstream $x$ and $y$ steering magnets.

For the next measurement, the second-order (quadrupole and skew) moments of the 0.9 and 8-nC electron beams depending on the rf phase of the booster klystron were measured while the beam positions of the electron beam were fixed at the center of the BESM with the beam-orbit and energy feedbacks on. Beam-orbit feedback using upstream steering magnets controlled the beam positions with a resolution of $0.1 \mathrm{~mm}$ at the exit of sector B in order to reduce the emittance growth by a transverse wakefield. The energy feedback controlled the beam-energy stability at the exit of sector B within a standard deviation of $0.03 \%$. The transverse spatial profiles of the beam were simultaneously measured by the screen-monitor system in order to calibrate the BESM results at a rate of $1 \mathrm{~Hz}$. One data point of the BESM was obtained by averaging 200 successive data with the statistical errors after the position dependence was corrected. The data of the screen monitor were obtained by averaging 50 successive measurements with the estimated errors.

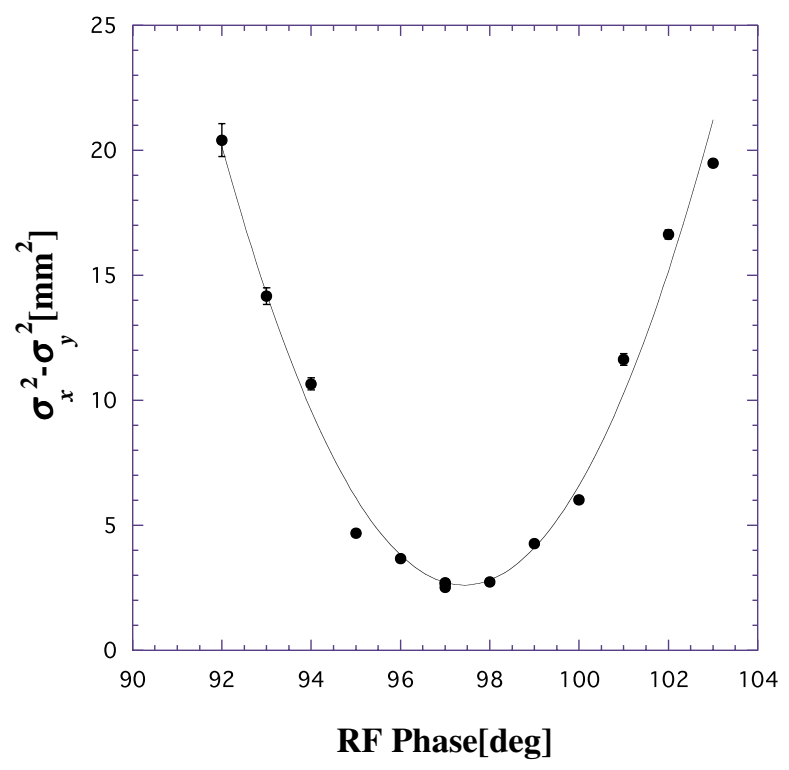

FIG. 4. (Color) Variations of the square difference of the transverse beamwidths (HWHM) for the 0.9-nC electron beam measured by the screen monitor. The solid line shows a curve with a quadric least-squares fitting procedure.

Figure 4 shows the result measured by the screen monitor depending on the rf phase $\phi$ of the booster klystron at sector $\mathrm{B}$.

\section{EXPERIMENTAL RESULTS}

\section{A. Second-order-moment analysis}

Figures 5(a) and 5(b) show that the variations of the quadrupole moment depending on the field strength, thus, the transverse beam positions, of (a) the horizontal and (b) vertical upstream steering magnets for the 0.9-nC electron beam with and without the beam-position corrections, respectively.

It is clearly shown that after the correction of the beam positions, the quadrupole moments are constant over the measured range of the field strength of the steering magnet within the estimated errors after correcting the beam positions where the quadrupole moments were corrected by following Eq. (12). This means that the quadrupole moment of the charged beam needs to be corrected by the transverse beam positions in order to extract the difference of the squared beamwidths.

Figure 6 shows the variations of the quadrupole moment for the 0.9 and 8-nC electron beams depending on the rf phase of the booster klystron, thus the transverse beamwidths, after the beam-position corrections.

For the 0.9-nC electron beam the beamwidth data directly measured by the screen monitor are also added in order to compare the BESM data after the beam-profile correction on the transverse distributions. The BESM data are related to the $\mathrm{SC}$ data by 
(a)

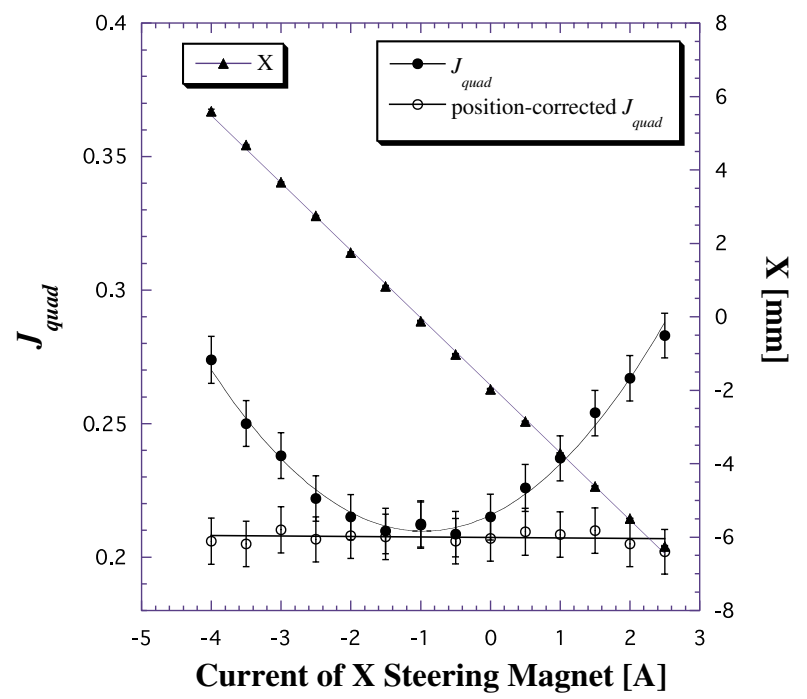

(b)

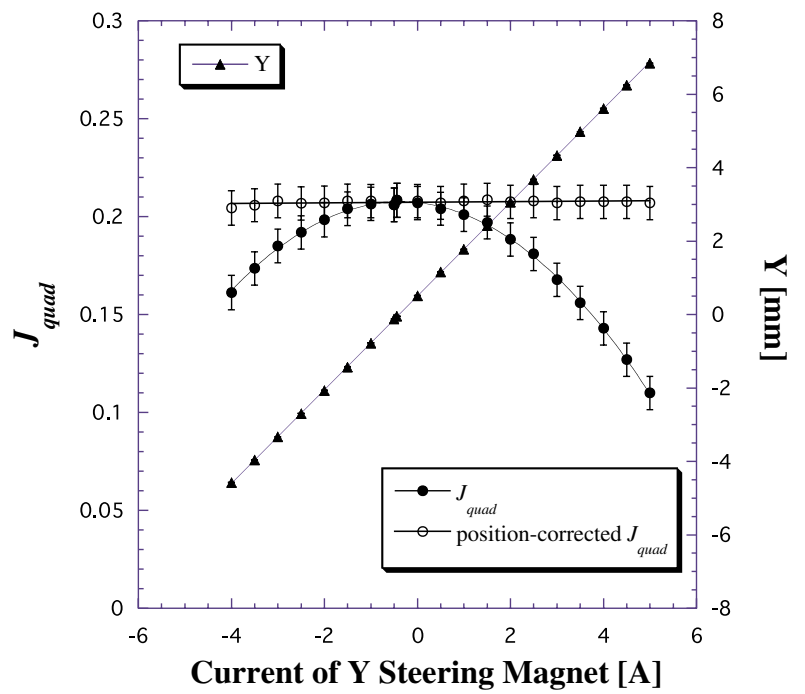

FIG. 5. (Color) Variations of the quadrupole moment depending on the field strength of (a) the horizontal and (b) the vertical upstream steering magnets for the 0.9-nC electron beam with and without beam-position corrections, respectively. The straight lines show that the beam positions are very linear with the field strength of the steering magnets.

$$
\left\langle x^{2}\right\rangle-\left\langle y^{2}\right\rangle=f\left(X^{2}-Y^{2}\right)+g,
$$

where $X$ and $Y$ are the transverse beamwidths measured by the screen monitor in HWHM, $g$ is a parameter mainly caused by the gain imbalance and the geometrical errors of the eight pickups of the BESM, and the parameter $f$ is the beam-profile correction factor depending upon the transverse distributions of the electron beam. If the transverse distribution can be described by a Gaussian function, the beam-profile correction factor is $f=1 / 1.18^{2}$. The calculation result with a quadric leastsquares fitting procedure gives the correction factor $f=$

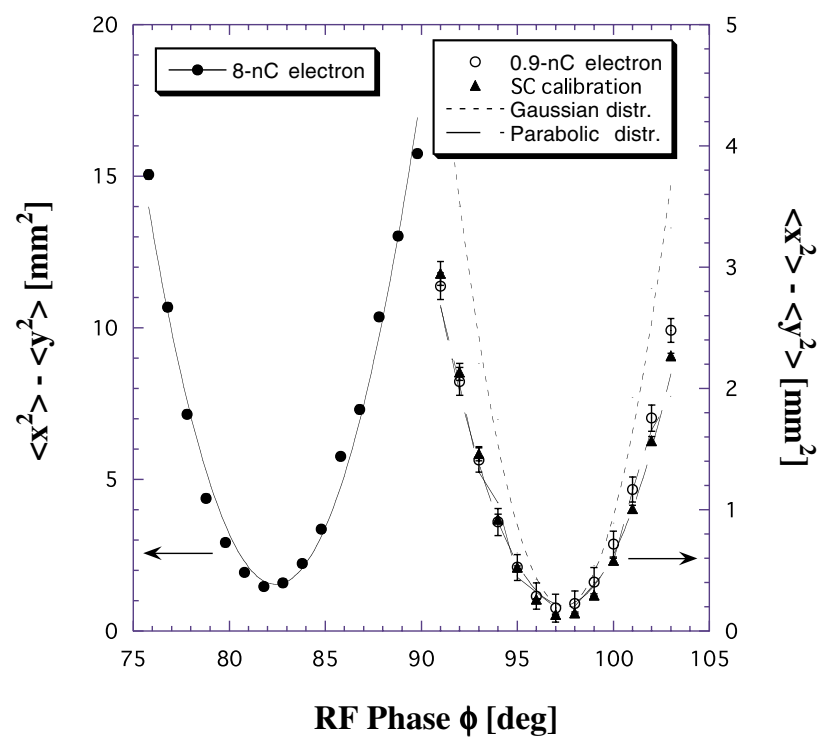

FIG. 6. (Color) Variations of the quadrupole moment for the 0.9 and the 8-nC electron beams depending on the rf phase of the booster klystron. The solid line for the $8-\mathrm{nC}$ electron beam shows a curve with a quadric least-squares fitting procedure. The other curves for the 0.9-nC electron beam show curves with a quadric least-squares fitting procedure assuming the transverse-distribution functions [see the text (Sec. VI A) for details].

$1 / 1.46^{2}$. This result shows that the transverse beam profile is approximately described not by a Gaussian function, but by a parabolic function. The BESM data agree well with the corrected SC data within the estimated errors over the measured region of the rf phase (see Fig. 6). On the other hand, for the 8-nC electron beam, although the transverse beam profiles were measured by the screen monitor, it is difficult to estimate the beamwidths from the beam-profile distributions, since the horizontal distribution was largely deformed by a longitudinal wakefield at the rf phase, apart from the phase which gave the energy-spread minimum. Figure 7 shows the relative $\mathrm{rf}$ phase resolutions obtained for both electron beams by the BESM. The result shows that the rf phase resolution for the $0.9-\mathrm{nC}$ electron beam is less than $1^{\circ}$ over the region of $\pm 1^{\circ}$ apart from the rf phase at the energy-spread minimum, while that for the 8-nC electron beam is less than $1^{\circ}$ over the whole measured region.

The skew angle $\left(\theta_{\text {skew }}\right)$ of the electron beam in the transverse plane can be derived by using Eqs. (12) and (13),

$$
\theta_{\text {skew }}=J_{s} / 2 J_{q}
$$

Figure 8 shows the variations of the skew angles depending on the rf phase of the booster klystron obtained for both electron beams after beam-position and offset corrections for the second-order moments. The average skew 


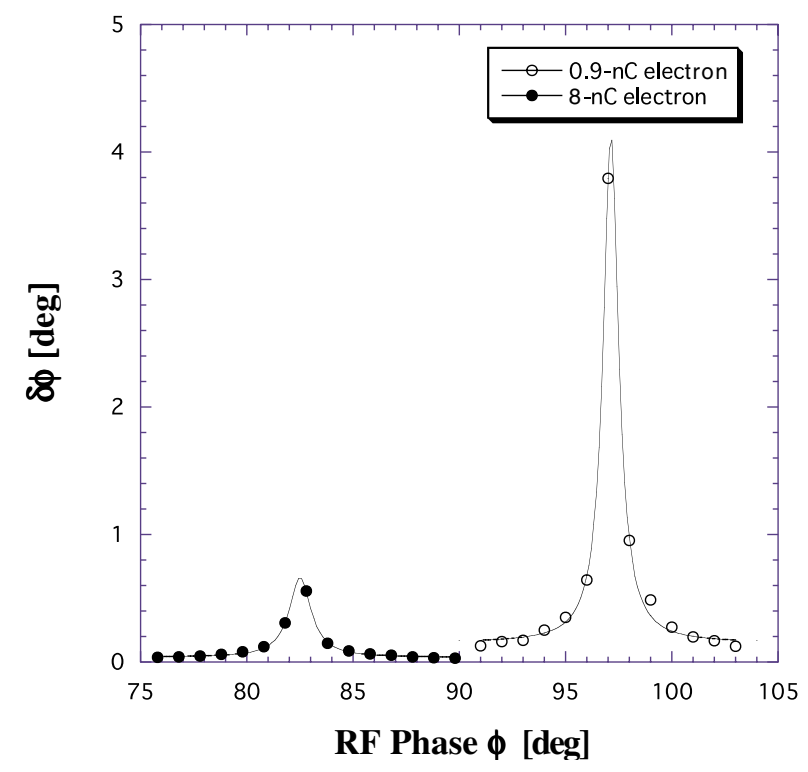

FIG. 7. (Color) Variations of the relative $\mathrm{rf}$ phase resolution obtained for the 0.9 and 8 -nC electron beams. The solid curves drawn through the data are only to guide the eye.

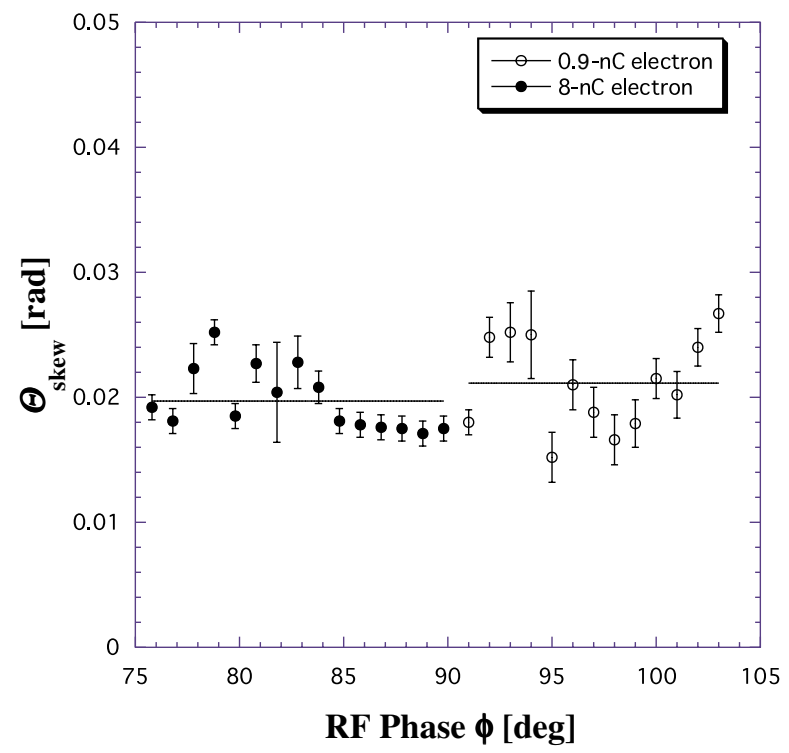

FIG. 8. (Color) Variations of the skew angles depending on the rf phase of the booster klystron obtained for the 0.9 and 8-nC electron beams. The solid lines show the average skew angles over the measured rf region.

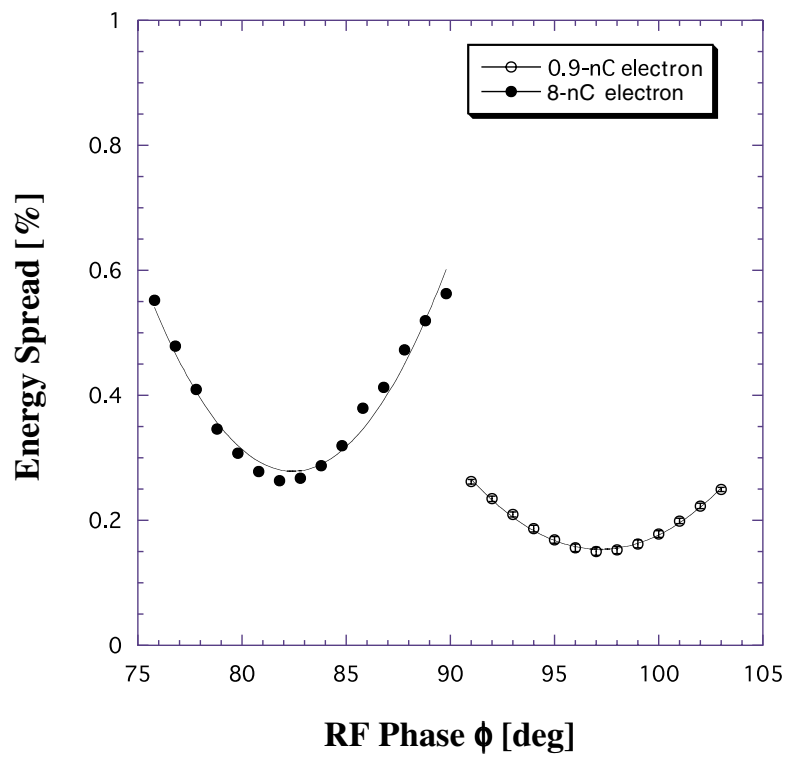

FIG. 9. (Color) Variations of the energy spread depending upon the rf phase of the booster klystron obtained for the 0.9 and 8$\mathrm{nC}$ electron beams. The solid lines show curves with a quadric least-squares fitting procedure.

angles are $21 \pm 4$ and $20 \pm 3 \mathrm{mrad}$ for the 0.9 and $8-\mathrm{nC}$ electron beams over the measured region of the rf phase, respectively.

\section{B. Energy-spread analysis}

Assuming that the vertical dispersion $\eta_{y}$ is zero at the BESM, the energy spread $\Delta E$ of the electron beam can be estimated from the transverse beamwidths along with the optics parameter ( $\beta$ functions and horizontal dispersion $\eta_{x}$ ) and the transverse emittances as follows:

$$
\left\langle x^{2}\right\rangle-\left\langle y^{2}\right\rangle \simeq \beta_{x} \epsilon_{x}+\left(\eta_{x} \Delta E / E\right)^{2}-\beta_{y} \epsilon_{y}+g,
$$

where $g$ is the same parameter as defined in Eq. (14), and the transverse emittances, $\epsilon_{x}$ and $\epsilon_{y}$, were measured by wire scanners at the end of sector B. The optics parameters and the emittances are summarized in Table II.

Figure 9 shows the energy spread obtained for both electron beams. The result shows that the obtained energy spreads are $0.150 \% \pm 0.007 \%$ and $0.264 \% \pm 0.004 \%$ for

TABLE II. Optics parameters and transverse emittances at the BESM.

\begin{tabular}{|c|c|c|c|}
\hline \multicolumn{4}{|l|}{ Optics parameter } \\
\hline$e^{-}$charge $(\mathrm{nC})$ & 0.9 & & 8 \\
\hline$\beta_{x} / \beta_{y}(\mathrm{~m})$ & & $2.56 / 9.30$ & \\
\hline$\eta_{x} / \eta_{y}(\mathrm{~m})$ & & $0.760 / 0$ & \\
\hline$\epsilon_{x} / \epsilon_{y}(\mathrm{~m})$ & $8.05 / 14.2 \times 10^{-8}$ & & $2.75 / 3.49 \times 10^{-7}$ \\
\hline
\end{tabular}


the electron beams with charges of 0.9 and $8 \mathrm{nC} /$ bunch at the rf phase of the energy-spread minimum, respectively. The resolution for the energy-spread measurement is on the order of $10^{-3}$, depending on the beam charge.

\section{CONCLUSIONS}

An energy-spread measurement for single-bunch electron beams using a nondestructive beam energy-spread monitor with multi-strip-line electrodes was successfully carried out at the KEKB injector linac. The analysis was performed based on a multipole-moment analysis of the electromagnetic field induced on the electrode surfaces. The second-order (quadrupole and skew) moments of electron beams with charges of 0.9 and $8 \mathrm{nC} /$ bunch were accurately measured by this monitor. The transverse beamwidths are consistent with those measured by the screen monitor within the estimated errors. The experimental result shows that the quadrupole moment derived from the analysis must be corrected in order to extract the difference of the squared beamwidths by the transverse beam positions. The average skew angles in the transverse plane are $21 \pm 4$ and $20 \pm 3 \mathrm{mrad}$ for the 0.9 and $8-\mathrm{nC}$ electron beams over the measured region of the rf phase. The result also shows that the analyzed energy spreads were $0.150 \% \pm 0.007 \%$ and $0.264 \% \pm 0.004 \%$ for electron beams with charges of 0.9 and $8 \mathrm{nC} /$ bunch at the $\mathrm{rf}$ phase of the energy-spread minimum, respectively. The principal advantage of this monitor over a wire scanner is that the energy spread can be nondestructively obtained on a pulse-by-pulse basis. The result shows that the $\mathrm{rf}$ phase resolution for the 0.9-nC electron beam was less than $1^{\circ}$ over the region of $\pm 1^{\circ}$ apart from the rf phase at the energy-spread minimum, while that for the 8-nC electron beam was less than $1^{\circ}$ over the whole measured region. These rf phase resolutions are sufficient to control the long-term drift of the energy spread of the electron beams at the $180^{\circ}$ arc in the injector linac. Software development for the energy-spread feedback system at the arc is in progress by applying a technique similar to the energy-feedback system used in daily operation.

\section{ACKNOWLEDGMENTS}

The authors would like to thank Professor K. Oide for his useful discussions of the multipole-moment analysis applied to the beam energy-spread monitor.
[1] S. Kurokawa and E. Kikutani, Nucl. Instrum. Methods Phys. Res., Sect. A 499, 1-7 (2003).

[2] I. Abe et al., Nucl. Instrum. Methods Phys. Res., Sect. A 499, 167-190 (2003).

[3] T. Suwada, N. Kamikubota, H. Fukuma, N. Akasaka, and H. Kobayashi, Nucl. Instrum. Methods Phys. Res., Sect. A 440, 307 (2000).

[4] K. Furukawa, A. Enomoto, N. Kamikubota, T. Kamitani, Y. Ogawa, S. Ohsawa, K. Oide, and T. Suwada, in Proceedings of the International Conference on Accelerator and Large Experimental Physics Control Systems (ICALEPCS'99), Trieste, Italy, 1999 (Elettra, Trieste, 1999), pp. 248-250.

[5] K. Furukawa, N. Kamikubota, T. Suwada, and T. Obata, in Proceedings of the International Conference on Accelerator and Large Experimental Physics Control Systems (ICALEPCS2001), San Jose, CA, 2001 (SLAC Report No. SLAC-R-592, eConf C011127), pp. 266-268.

[6] T. Suwada, A. Enomoto, T. Urano, and H. Kobayashi, in Proceedings of the 20th Linear Accelerator Meeting in Japan, Osaka, 1995 (FELI, Osaka, 1995), pp. 245-247.

[7] N. Iida, Y. Funakoshi, M. Kikuchi, K. Satoh, T. Suwada, and T. Kawamoto, in Proceedings of the 1999 Particle Accelerator Conference (PAC'99), New York City, NY, 1999 (IEEE, Piscataway, NJ, 1999), pp. 2108-2010.

[8] R. H. Miller, J.E. Clendenin, M. B. James, and J.C. Sheppard, in Proceedings of the 12th International Conference of High-Energy Accelerators (HEAC'83), Fermilab, 1983 (Fermilab, Illinois, 1983), pp. 602-605.

[9] T. Suwada, Jpn. J. Appl. Phys. 40, 890-897 (2001).

[10] T. Suwada, in Proceedings of the the XXth International Linac Conference (LINAC2000), Monterey, California, 2000 (SLAC Report No. SLAC-R-561, eConf C000821), pp. 199-201.

[11] J. D. Jackson, Classical Electrodynamics (John Wiley \& Sons, New York, 1975), 2nd ed., p. 54.

[12] S. Ohsawa, J.-Y. Choi, Y. Ogawa, A. Enomoto, T. Kamitani, H. Kobayashi, and I. Sato, in Proceedings of the XVIII International LINAC Conference (LINAC'96), CERN, Geneva, Switzerland, 1996 (CERN Report No. 96-07), pp. 815-817.

[13] Y. Ogawa, K. Furukawa, T. Kamitani, S. Ohsawa, A. Enomoto, T. Suzuki, S. Abe, and K. Iwasaki, in Proceedings of the First Asian Particle Accelerator Conference (APAC'98), KEK, Tsukuba, Japan, 1998 (KEK Report No. 98-10), pp. 534-536.

[14] T. Kamitani et al., in Proceedings of the First Asian Particle Accelerator Conference (APAC'98), KEK, Tsukuba, Japan, 1998 (Ref. [13]), pp. 429-431. 